\title{
Root Resorption of Central Incisor by Impacted Canine in Unusual Transposition: Orthodontics and Implant Rehabilitation: 3-Year Follow-Up
}

\author{
Miguel Ángel Garcés-Villalá1 ${ }^{*}$, Silvia Lorena Sticca-Peralta ${ }^{1}$, Sergio David Rico-Jordán', \\ José Luis Calvo-Guirado² \\ ${ }^{1}$ Department of Implant and Biomaterial Research, Fundación Corazón de Jesús, San Juan, Argentina \\ ${ }^{2}$ Department of Oral Surgery and Implant Dentistry, Faculty of Health Sciences, Universidad Católica San Antonio de Murcia, \\ Murcia, España \\ Email: ^fundacioncorazondejesus@gmail.com, lorenasticca@hotmail.com, davidricoodonto@yahoo.com.ar, jlcalvo@ucam.edu
}

How to cite this paper: Garcés-Villalá, M.Á., Sticca-Peralta, S.L., Rico-Jordán, S.D. and Calvo-Guirado, J.L. (2021) Root Resorption of Central Incisor by Impacted Canine in Unusual Transposition: Orthodontics and Implant Rehabilitation: 3-Year Follow-Up. Journal of Biosciences and Medicines, 9, 55-64.

https://doi.org/10.4236/jbm.2021.910005

Received: September 13, 2021

Accepted: October 11, 2021

Published: October 14, 2021

Copyright $\odot 2021$ by author(s) and Scientific Research Publishing Inc. This work is licensed under the Creative Commons Attribution International License (CC BY 4.0).

http://creativecommons.org/licenses/by/4.0/

\section{(c) (i) Open Access}

\begin{abstract}
This case presents an unusual transposition of an impacted canine on the central incisor that provides a multidisciplinary approach to its resolution. The patient comes to the consultation for mobility of the central incisor. Clinically, tooth mobility was confirmed with tooth crowding and persistence of the temporary canine. Cone beam tomography (CBCT) observed the atypical position of the permanent canine that caused root resorption of the ipsilateral central incisor and the need to extract both teeth. The conservative treatment of the bone structures during the dental enucleation of the impacted canine carried out by odontosection and the immediate bone grafting of the defect projected an acceptable regeneration of the bone volume for the insertion of the implant. The orthodontic treatment managed to correct the bite, position the upper premolar in the place of the canine tooth and maintain the space to place the implant in the central incisor. The three-year follow-up shows maintenance of the peri-implant crestal bone level, the success of orthodontic treatment with recovery of the patient's function and aesthetics.
\end{abstract}

\section{Keywords}

Impacted Canine, Unusual Transposition, Root Resorption, Orthodontics/Dental Implants

\section{Introduction}

The case presented is unique because it presents an impacted canine in the un- 
usual position of the central incisor to which half of its root was reabsorbed. Excluding the third molars, the maxillary canine is the most commonly impacted tooth followed by the mandibular canines. According to Al-Zoubi et al. given the complexities, impacted canines can have a wide range of variations in their long axis and should be carefully evaluated during treatment planning [1].

Peck et al. define dental transposition as the change of position of 2 adjacent teeth or not, especially in relation to their roots in the same quadrant of the dental arch, which develops and erupts in inverted positions and alters the normal sequence of the eruption [2].

Becker and Chaushu found that dental transpositions have a relatively low prevalence in the population, approximately $0.1 \%$ to $0.4 \%$ in both jaws. They are generally unilateral, more frequent in the upper arch between $70 \%$ and $80 \%$ and between canines and premolars. Furthermore, they state that when an impacted permanent maxillary canine is diagnosed and a malocclusion exists, a full orthodontic evaluation is needed for overall planning. The orthodontist will perform a clinical examination. When all the information is gathered, a treatment plan will be formulated to resolve the general malocclusion, which may lead to the decision to extract the permanent teeth [3].

It is quite clear that the greatest risk is the possibility of root resorption in the adjacent teeth. Studies using Cone Beam computed tomography (CBCT) indicate that the percentage of root resorption in lateral incisors caused by impacted canines ranges from 38\% for Ericson and Kurol [4] to 66.7\% reported by Walker et al. [5].

Grisar et al. advise that treatment planning for the impacted maxillary canine should be based on 3D images. With CBCT, it is possible to correctly define the position of the impacted maxillary canine and recognize accompanying anomalies such as ankylosis, root dilaceration with or without anchoring to the floor of the sinus or nasal cavity, resorption of neighboring teeth or odontoma [6]. Becker et al. studied the influence of age on impacted teeth, claiming that they are more difficult to treat in adults, the success rate among patients older than 30 years was $41 \%$, while the success rate for those of 20 at 30 years was $100 \%$ [7]. The unusual transposition of the impacted canine leads to the loss of two fundamental teeth for the esthetics and function of the stomatognathic system. The objective of our interdisciplinary approach work is to present a predictable treatment plan to rehabilitate normal dental anatomy, position and occlusion through synchronous orthodontic and implantology treatment.

\section{Case Report}

A 39-year-old healthy female patient without comorbidities presented to the consultation. The main complaint of the patient is the dental mobility of the central incisor that, in addition to hindering the masticatory function, puts aesthetics at risk. For fear of surgery, she postponed her consultation and no previous treatments were performed. 


\subsection{Clinical Findings}

The patient has a mesofacial biotype, with a straight profile. Transversally both jaws of her present compression, causing a cross bite from canine to second molar on the left side. The midlines do not match and the highlight and criss-cross above are minimal. On the right side she presents molar and canine class I and molar and canine class III on the left side (Figure 1).

\subsection{Timeline}

\begin{tabular}{|c|c|c|c|c|c|c|}
\hline $\begin{array}{c}\text { Interdisciplinary } \\
\text { consultation }\end{array}$ & Diagnosis & $\begin{array}{l}\text { Treatment } \\
\text { plan }\end{array}$ & $\begin{array}{c}\text { Tooth } \\
\text { extractions }\end{array}$ & Orthodontics & $\begin{array}{l}\text { Dental } \\
\text { implant }\end{array}$ & Follow-up \\
\hline
\end{tabular}

Teamwork allows an interdisciplinary precision diagnosis through different cephalometric and tomographic studies. A synchronous orthodontic and implantology treatment plan diagrammed on a timeline is key to determining the opportune moments of intervention of each component professional discipline and achieving a successful final result.

\subsection{Diagnostic Evaluation}

The first diagnostic imaging method is panoramic radiography or orthopantomography (Figure 2(a)), which allows a general evaluation of the pathologies and their relationship with adjacent anatomical structures. The diagnostic challenge in this case was to determine the exact three-dimensional position of the impacted tooth, for which cone beam tomography (CBCT) was used. The upper left canine is observed in total bone retention with its incisal edge located in the

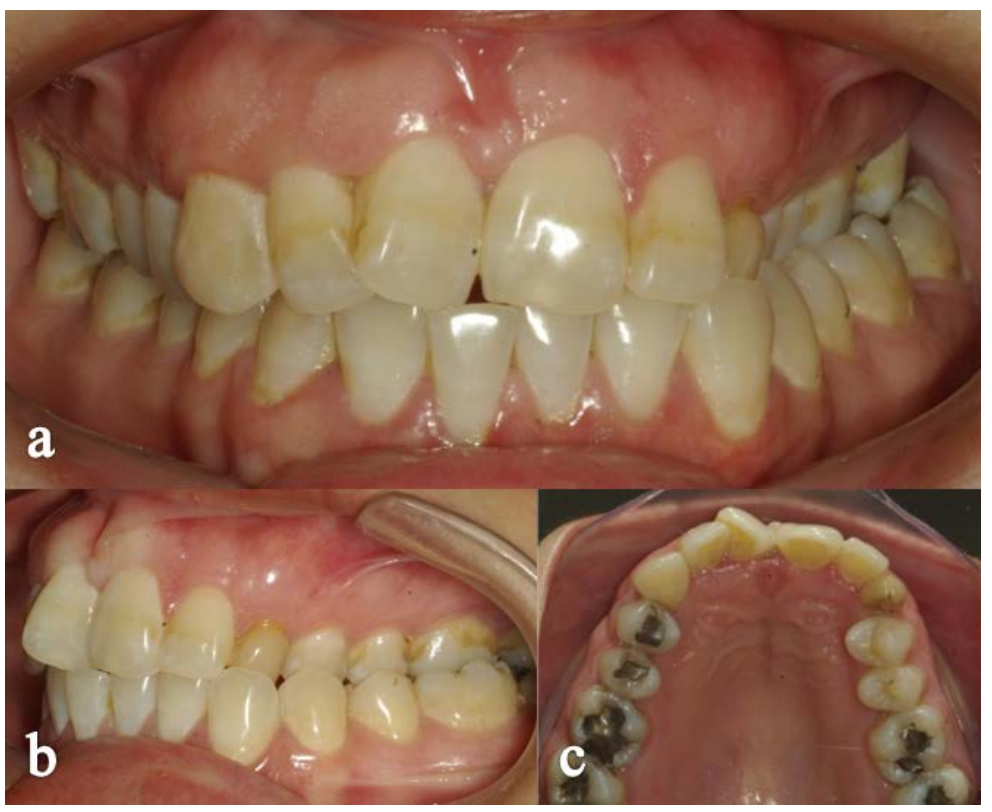

Figure 1. (a) and (b) Clinical view shows a central incisor in gyversion and inverted bite on the left side. (c) Clinical occlusal view of the maxilla, showing tooth crowding and persistence of the upper left temporary canine. 


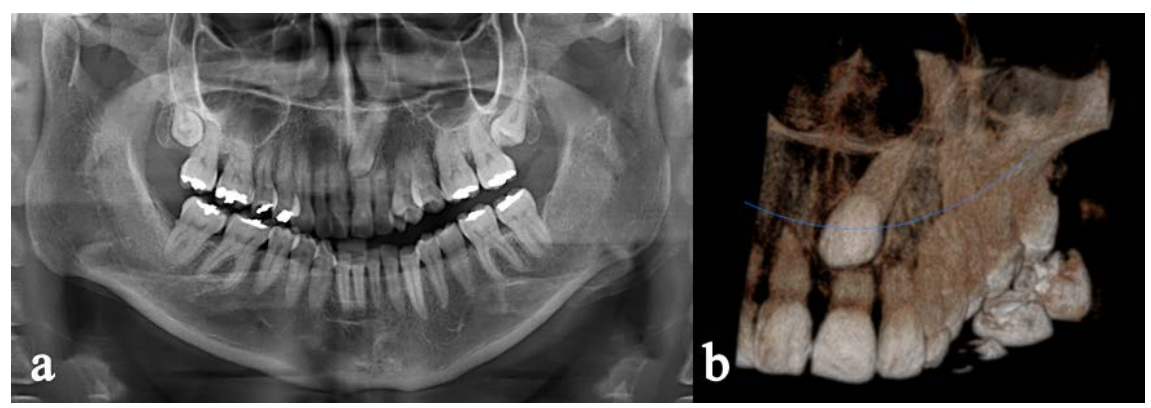

Figure 2. (a) Orthopantomography. (b) Three-dimensional reconstruction with cone beam tomography $(\mathrm{CBCT})$. The canine in retention and the resorption that it caused in the ipsilateral central incisor is observed.

middle third of the root of the upper left central incisor that presents root resorption and tooth mobility grade 2 . The apex of the impacted canine is in intimate contact with the floor of nostrils (Figure 2(b)).

Using the models of the jaws mounted on the articulator, frontal and profile photographs, the orthodontic treatment plan was initiated using the lateral cranial X-ray of the patient to perform the cephalometric study and determine the treatment plan (Figure 3(a), Figure 3(b)).

\section{Therapeutic Intervention}

The equations In the planning of the treatment (interdisciplinary) and prior to orthodontic treatment, in the initial stage it was decided to perform the simultaneous enucleation of the two impacted canine teeth and central incisor with root resorption mobility and poor prognosis. Digital radiovisiography (RVG) with a Kodak 5100 radiovisiograph was used to control the sequence of the surgical procedure (Figure 4).

After infiltrative anesthesia with $4 \%$ articaine, a simple extraction of the incisor was performed and a mucoperiosteal flap was detached in the anterior area of the palate. Using the post-extraction socket as access, the dental canine crown was sectioned (Figure 4(b)). The root portion was dislocated and extracted with a straight elevator, and the cavity toilet was completed with a molt curette (Figure $4(\mathrm{c})$ ). The bone defect was filled (Figure 4(d)) with a synthetic bone graft from Argentinean beta tricalcium phosphate ceramic developed by Garces Villalá et al. [8].

\subsection{Orthodontic Treatment}

In addition to the extraction of the central incisor and impacted canine, the upper right temporary canine and the first lower premolars were extracted to achieve class I on both sides. The MBT Straight Arch Technique is used and with a sequence of arches (Figure 5(a)) alignment and balancing of both arches is achieved, criss-Cross type rubber is used from palatal 25 and 26 to vestibular 35 and 36 to reverse the left cross bite. The central incisor space is maintained and then the dental implant is placed (Figure 5(b)), tooth 24 is mesialized to occupy 


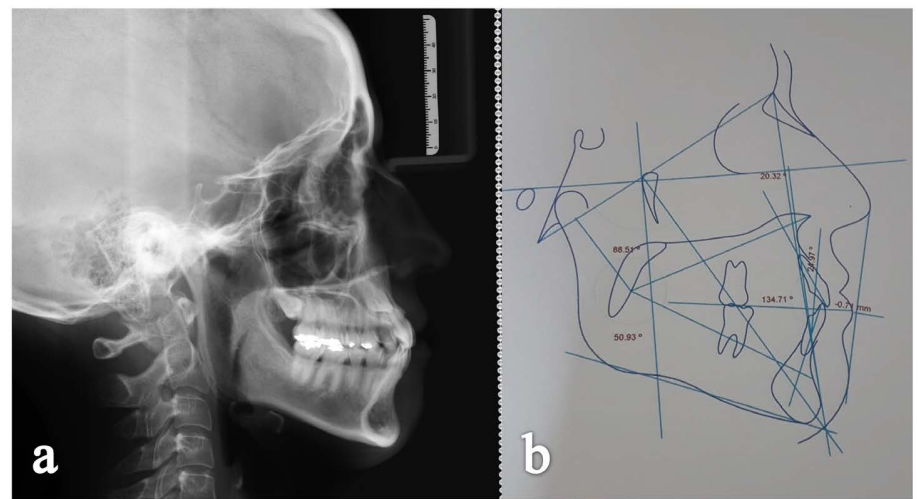

Figure 3. (a) Lateral cranial X-ray. (b) Cephalometry.

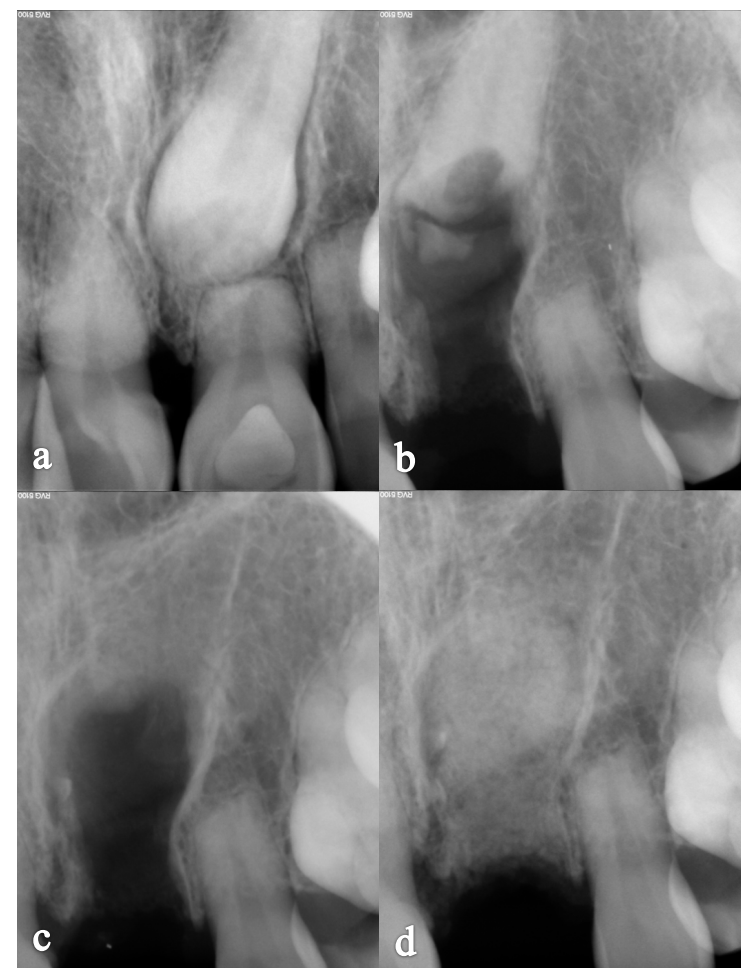

Figure 4. RVG of the surgical sequence of the extraction: (a) Preoperative X-ray, (b) Extraction of the central incisor and dental section of the dental crown of the retained canine, (c) surgical bed after dental extractions and (d) filling of the bone defect with the graft osseous.

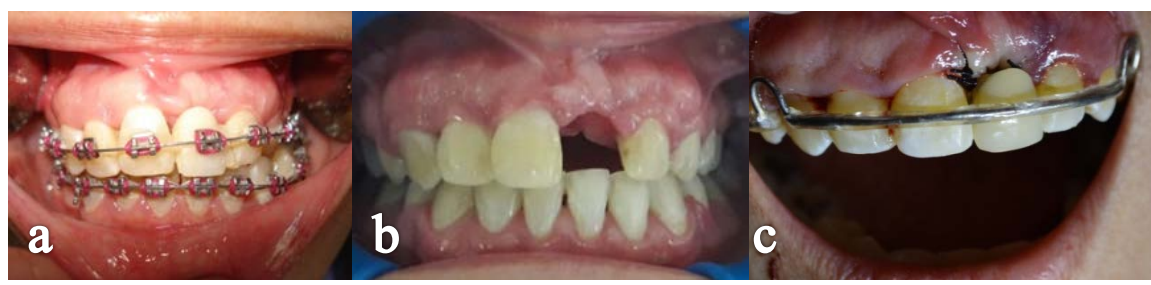

Figure 5. (a) Sequence of archwires, with stock tooth fixed to the archwire to maintain tooth 21 space. (b) Orthodontics completed, with the place ready to receive implant. (c) Hawley plate with integral archwire and tooth 21 , to give esthetics and containment, which is placed on the day of implant surgery. 
the place of 23 and thus achieve left canine class I. Correct highlighting and criss-crossing and midline coincidence are also achieved. At the end of the treatment, a fixed lower retention 3-3 and a Hawley plate with an integral arch were placed, to which tooth 21 was placed to maintain the space until the implant was placed (Figure $5(\mathrm{c})$ ).

\subsection{Implantological Treatment}

After the bone graft had healed (Figure 6(a) and Figure 7(a)), the insertion surgery of the hexagonal internal connection dental implant was performed (Figure 6(b) and Figure 6(c)). The access was made with an incision towards the palatine (Figure $7(\mathrm{~b})$ ) and an upper labial frenectomy was also performed (Figure 7(c)) to prevent gingival recession on the future dental crown of the implant. After four months of hard and soft tissue healing (Figure $7(\mathrm{~d})$ ), a gingival healing abutment (Figure $7(\mathrm{e})$ ) is connected, allowing soft tissue stability after four weeks (Figure 7(f)). At this time, the impression is taken with regular silicone, a transfer is used and its adjustment is controlled radiographically (Figure 6(d)). On plaster models, a metal casting is made, its fit (Figure 6(e)) and dental occlusion (Figure $7(\mathrm{~g})$ ) are checked, which will support the ceramic of the finished prosthesis (Figure 6(f) and Figure 7(h)).

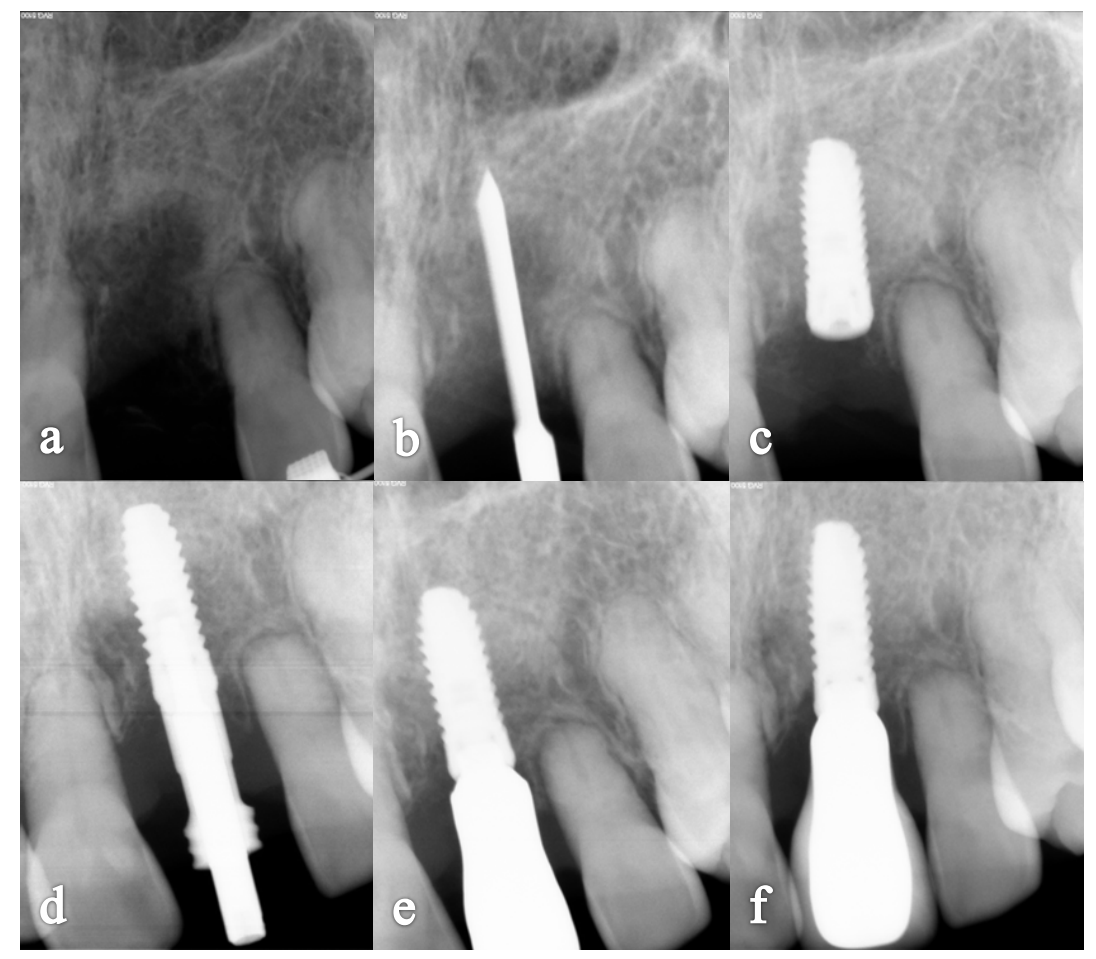

Figure 6. RVG of the surgical-prosthetic sequence of the dental implant: (a) Preoperative $\mathrm{X}$-ray after 4 months of bone graft healing, (b) X-ray with a lance drill to check the mesiodistal implant position, (c) Hexagonal internal connection implant in ideal position, (d) $\mathrm{Rx}$ control of impression adjustment with transfer after 4 months of osseointegration of the implant, (e) Rx test of adjustment of the metal casting and (f) Implant and metal-ceramic dental crown completed. 


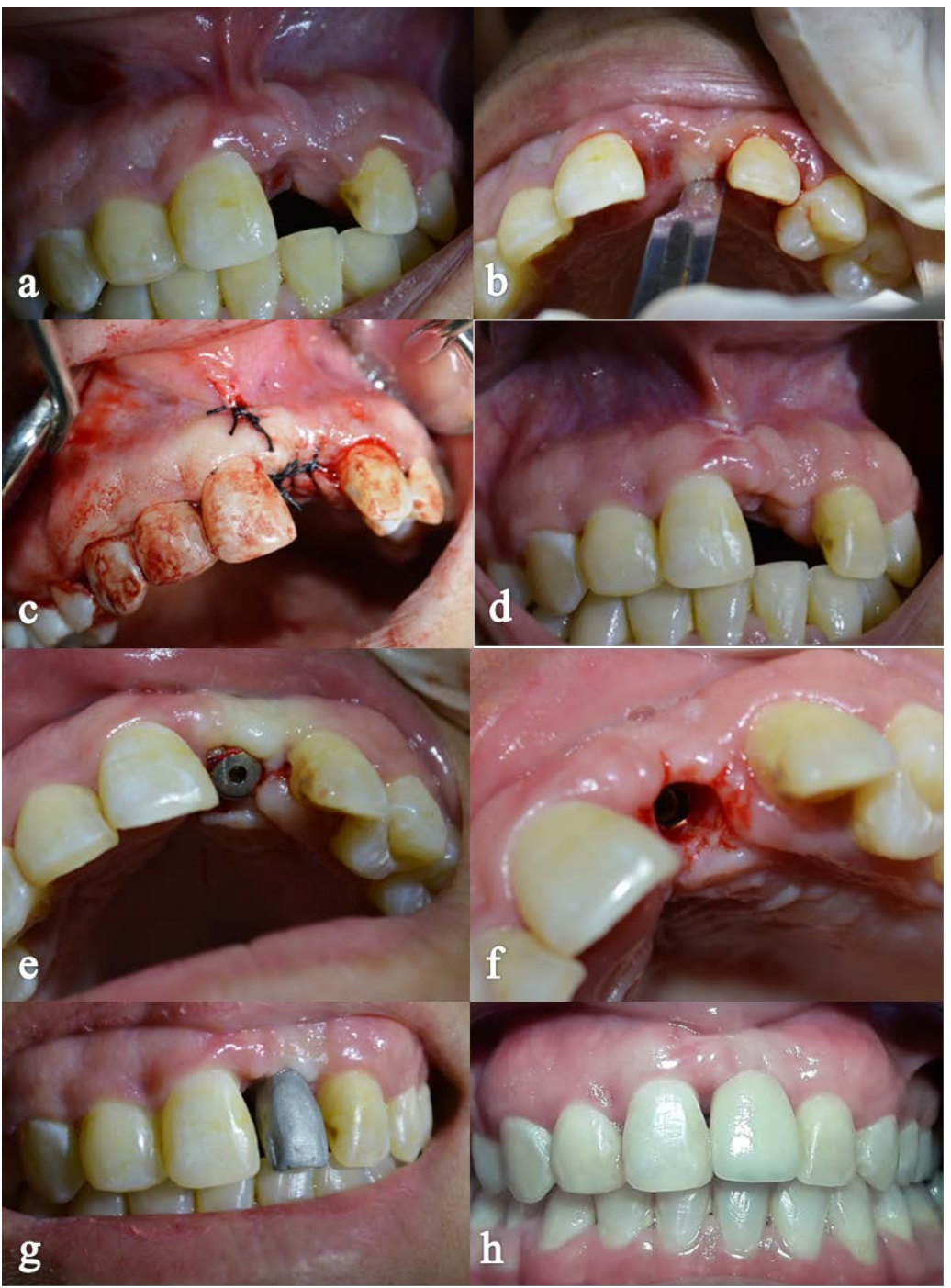

Figure 7. (a) Bone graft healing at 4 months, (b) incision with blade No. 15 towards the palatine, (c) Frenectomy and insertion of the implant, (d) healing at 4 months, (e) Connection of the healing abutment, ( $\mathrm{f}$ ) view of the implant, (g) cast metal trial, h) adjustment of the completed dental crown.

The patient was summoned for six-monthly check-ups during the first three years, in which a check-up of the occlusion/maintenance of the position of the dental elements and stability of the dental implant crown was performed, and then continued with annual check-ups.

\section{Monitoring and Results}

The interdisciplinary treatment added to the fundamental collaboration of the patient managed in two years of treatment to restore functional and aesthetic harmony and satisfy his expectations. To measure the marginal bone loss of the implant over time, we used CBCT and RVG. Photographs were used to evaluate the stability of the peri-implant soft tissues and to control orthodontic treatment. The patient collaborated throughout the process. There were no adverse 
effects during the process. However, an unforeseen event occurred: a week after the implant insertion surgery, a seed from the patient's diet entered the palatine mucoperiosteum causing a foreign body infection. It was medicated with a broad-spectrum antibiotic and the seed was extracted. In any case, the infection caused bone loss of $1.5 \mathrm{~mm}$ in the mid-palatal area of the implant neck. A three-year follow-up of treatment can be seen in Figure 8.

\section{Discussion}

In the present case, a possibility of comprehensive multidisciplinary treatment of an unusual dental transposition is presented, respecting biological and biomechanical criteria. Miranda Candeiro et al. affirm that dental transpositions are more frequent in the female sex and the left side is generally the most affected [9], which is consistent with the present case. The limitations observed in our work were the lack of regular follow-up (quarterly controls were avoided due to the COVID 19 pandemic), in addition, the unforeseen event of foreign body infection (seed) which modified the initially available bone volume.

Mithridade et al. suggest treatment alternatives other than the one proposed, such as the placement of implants through impacted teeth to avoid invasive surgery [10], however its routine application is not justified due to the lack of patients with long-term follow-up. Another possible treatment approach chosen by Cruz consists of orthodontic traction of the impacted canine and its repositioning [11] but in our case, it would have a poor prognosis because the impaction is very deep with marked angulation and the root of the canine is fully formed.

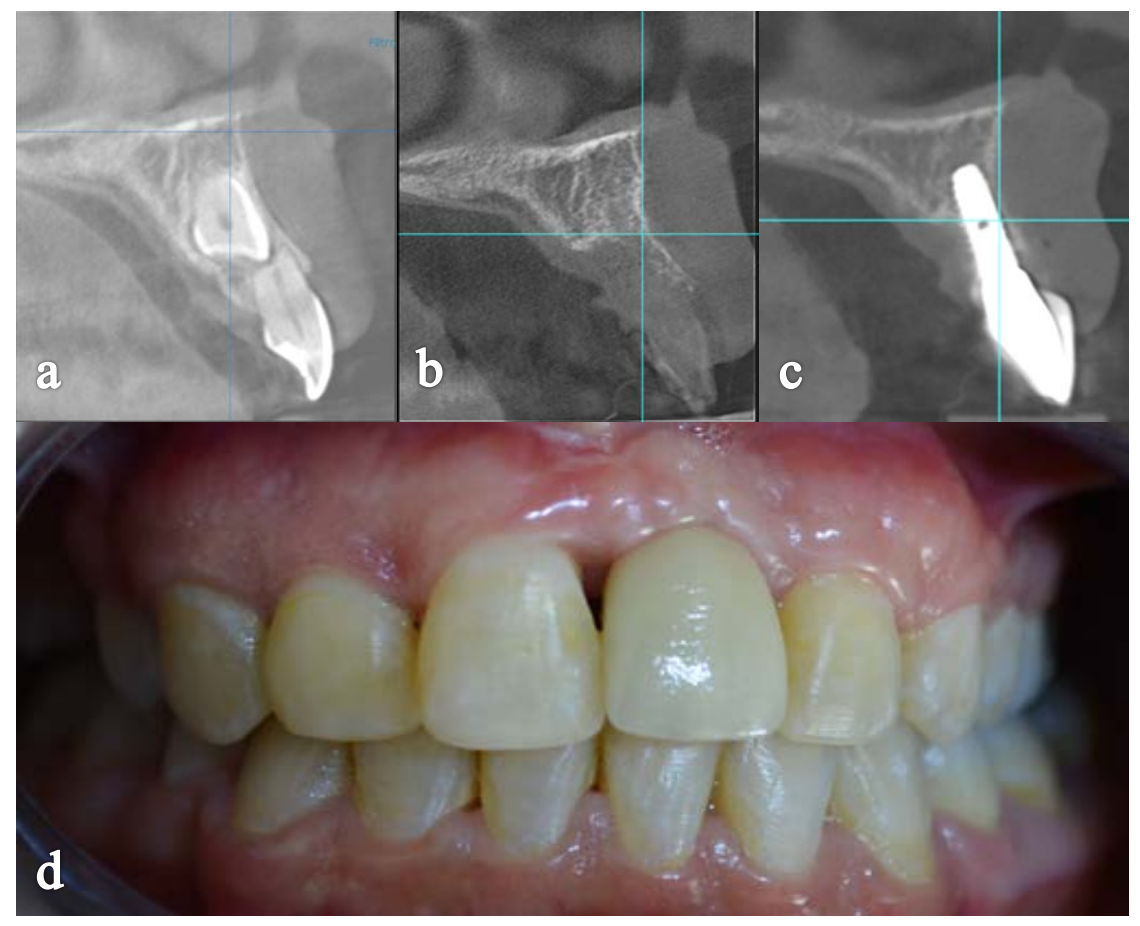

Figure 8. CBCT timeline (a) Preoperative CBCT, (b) CBCT 4 months healing of the graft, (c) CBCT Three years of dental implant follow-up and (d) Clinical vision at three years. 
Furthermore, as stated by Manne et al. the unusual position of the canine on the central incisor is very unfavorable and its orthodontic displacement could damage other teeth [12]. For this reason, we consider that our treatment plan, although it performs the dental extraction of the impacted canine which could be tractioned by orthodontics, preserves the integrity of the neighboring teeth. Yan et al. [13] published that maxillary canine impaction increases the risk of root resorption at adjacent teeth (incisors and first premolars). Physical proximity ( $\backslash 1$ $\mathrm{mm}$ ) between the impacted canine and an adjacent root is the most important predictor for root resorption. This situation was verified in our case, on the upper central incisor with a poor prognosis due to root resorption that had to be extracted (regardless of the chosen treatment plan) and rehabilitated by means of a dental implant.

\section{Conclusion}

The planning and synchronization of the interdisciplinary team is key in the success of the treatment of dental retentions of impacted upper canines. The atypical position of the canine caused root resorption of the central incisor and the need to extract both teeth. The orthodontic treatment managed to correct the bite, position the upper premolar in the place of the canine tooth and maintain the space to place the incisor dental implant. In addition, the conservative treatment of the bone structures during the dental enucleation surgery of the impacted canine, performed by dental section, achieved an acceptable regeneration of the bone volume through the bone graft to allow the insertion of the dental implant. The three-year follow-up confirmed the stability of the peri-implant bone structures and the maintenance of dental normo-occlusion. However, a longer follow-up period is needed to validate its routine application.

\section{Patient Perspective}

The patient favorably evaluated the treatment and highlighted the emotional support provided by the professional team, especially during the COVID-19 pandemic.

\section{Informed Consent}

The patient accepted the proposed treatment and signed the corresponding informed consent.

\section{Conflicts of Interest}

The authors declare no conflicts of interest regarding the publication of this paper.

\section{References}

[1] Al-Zoubi, H., Alharbi, A.A., Ferguson, D.J. and Zafar, M.S. (2017) Frequency of Impacted Teeth and Categorization of Impacted Canines: A Retrospective Radio- 
graphic Study Using Orthopantomograms. European Journal of Dentistry, 11, 117-121. https://doi.org/10.4103/ejd.ejd_308_16

[2] Peck, L., Peck, S. and Attia, Y. (1993) Maxillary Canine-First Premolar Transposition, Associated Dental Anomalies and Genetic Basis. Angle Orthod Appleton, 63, 99-109.

[3] Becker, A. and Chaushu, S. (2015) Surgical Treatment of Impacted Canines: What the Orthodontist Would Like the Surgeon to Know. Oral and Maxillofacial Surgery Clinics of North America, 27, 449-458. https://doi.org/10.1016/j.coms.2015.04.007

[4] Ericson, S. and Kurol, P.J. (2000) Resorption of Incisors after Ectopic Eruption of Maxillary Canines a CT Study. The Angle Orthodontist, 70, 415-423.

[5] Walker, L., Enciso, R. and Mah, J. (2005) Three-Dimensional Localization of Maxillary Canines with Cone-Beam Computed Tomography. American Journal of Orthodontics and Dentofacial Orthopedics, 128, 418-423. https://doi.org/10.1016/j.ajodo.2004.04.033

[6] Grisar, K., Piccart, F., Al-Rimawi, A.S., Basso, I., Politis, C. and Jacobs, R. (2019) Three-Dimensional Position of Impacted Maxillary Canines: Prevalence, Associated Pathology and Introduction to a New Classification System. Clinical and Experimental Dental Research, 5, 19-25. https://doi.org/10.1002/cre2.151

[7] Becker, A., Chaushu, G. and Chaushu, S. (2010) Analysis of Failure in the Treatment of Impacted Maxillary Canines. American Journal of Orthodontics and Dentofacial Orthopedics, 137, 743-754. https://doi.org/10.1016/j.ajodo.2008.07.022

[8] Garcés-Villalá, M., Rico, S., Nazar, S., Escudero-Pinel, M., Galván-Josa, V. and Calvo-Guirado, J. (2020) Evaluation of Two Highly Porous Microcrystalline Biphasic Calcium Phosphate-Based Bone Grafts for Bone Regeneration: An Experimental Study in Rabbits. Journal of Materials Science and Chemical Engineering, 8, 8-30. https://doi.org/10.4236/msce.2020.86002

[9] Miranda Candeiro, G.T., Campelo, F. and Robles Ruiz, J.J. (2011) Transposición dentaria del canino superior derecho a propósito de 3 casos. Revista Cubana de Estomatología, 48, 389-395.

[10] Mithridade, D., Serge, S.M., Keyvan, D., Nedjoua, C.O., Georgy, D. and Philippe, R. (2015) Unconventional Implant Placement IV. Implant Placement through Impacted Teeth to Avoid Invasive Surgery. Long-Term Results of 3 Cases. The Open Dentistry Journal, 9, 15-20. https://doi.org/10.2174/1874210601509010015

[11] Cruz, R.M. (2019) Orthodontic Traction of Impacted Canines: Concepts and Clinical Application. Dental Press Journal of Orthodontics, 24, 74-87. https://doi.org/10.1590/2177-6709.24.1.074-087.bbo

[12] Manne, R., Gandikota, C., Juvvadi, S.R., Rama, H.R.M. and Anche, S. (2012) Impacted Canines: Etiology, Diagnosis, and Orthodontic Management. Journal of Pharmacy \& BioAllied Sciences, 4, 234-238. https://doi.org/10.4103/0975-7406.100216

[13] Yan, B., Sun, Z., Fields, H. and Wang, L. (2015) La canine maxillaire incluse augmente le risque de résorption radiculaire des dents adjacentes: Un problème de proximité anatomique. L'Orthodontie Française, 86, 169-179.

https://doi.org/10.1051/orthodfr/2015014 